\title{
Mode transition of plasma expansion for laser induced breakdown in Air
}

Kohei Shimamura, Kohei Matsui, Joseph A. Ofosu, Ippei Yokota, and Kimiya Komurasaki

Citation: Appl. Phys. Lett. 110, 134104 (2017); doi: 10.1063/1.4979646

View online: https://doi.org/10.1063/1.4979646

View Table of Contents: http://aip.scitation.org/toc/apl/110/13

Published by the American Institute of Physics

\section{Articles you may be interested in}

Influence of gas flow velocity on the transport of chemical species in an atmospheric pressure air plasma discharge

Applied Physics Letters 110, 134102 (2017); 10.1063/1.4979178

Tutorial: Physics and modeling of Hall thrusters

Journal of Applied Physics 121, 011101 (2017); 10.1063/1.4972269

Emission enhancement of underwater collinear dual-pulse laser-induced breakdown spectroscopy with the second pulse defocused

Applied Physics Letters 110, 101102 (2017); 10.1063/1.4977893

Plasma filamentation and shock wave enhancement in microwave rockets by combining low-frequency microwaves with external magnetic field

Journal of Applied Physics 120, 063303 (2016); 10.1063/1.4960805

Enhanced electrohydrodynamic force generation in a two-stroke cycle dielectric-barrier-discharge plasma actuator

Applied Physics Letters 110, 194101 (2017); 10.1063/1.4983370

Experimental characterization of active plasma lensing for electron beams

Applied Physics Letters 110, 104101 (2017); 10.1063/1.4977894

\section{PHYSICS TODAY}

MANACER'S GUIDE

Accelerate R\&D with

Multiphysics Simulation
READ NOW

PRESENTED BY

$\subset \subset$ COSOL 


\title{
Mode transition of plasma expansion for laser induced breakdown in Air
}

\author{
Kohei Shimamura, ${ }^{1}$ Kohei Matsui, ${ }^{2}$ Joseph A. Ofosu, ${ }^{3}$ Ippei Yokota, ${ }^{1}$ and Kimiya Komurasaki ${ }^{2}$ \\ ${ }^{1}$ Department of Engineering Mechanics and Energy, University of Tsukuba, 1-1-1 Tennnodai, \\ Tsukuba Ibaraki 305-8573, Japan \\ ${ }^{2}$ Department of Aeronautics and Astronautics, The University of Tokyo, 7-3-1 Hongo, Bunkyo, \\ Tokyo 113-8656, Japan \\ ${ }^{3}$ Department of Advanced Energy, The University of Tokyo, 5-1-5 Kashiwa-no-ha, Kashiwa, Chiba 277-8561, \\ Japan
}

(Received 5 February 2017; accepted 21 March 2017; published online 31 March 2017)

\begin{abstract}
High-speed shadowgraph visualization experiments conducted using a $10 \mathrm{~J}$ pulse transversely excited atmospheric (TEA) $\mathrm{CO}_{2}$ laser in ambient air provided a state transition from overdriven to Chapman-Jouguet in the laser-supported detonation regime. At the state transition, the propagation velocity of the laser-supported detonation wave and the threshold laser intensity were $10 \mathrm{~km} / \mathrm{s}$ and $10^{11} \mathrm{~W} / \mathrm{m}^{2}$, respectively. State transition information, such as the photoionization caused by plasma UV radiation, of the avalanche ionization ahead of the ionization wave front can be elucidated from examination of the source seed electrons. Published by AIP Publishing. [http://dx.doi.org/10.1063/1.4979646]
\end{abstract}

A laser-induced plasma in a gaseous form has attracted great interest for use in engineering applications (e.g., laser ignition and space propulsion) and for analysis of solids (e.g., metals and geological samples). ${ }^{1,2}$ Elucidation of plasma parameters and gas dynamics in gaseous media is crucially important for improving the performance of applications and analysis. Gas breakdown is initiated near the focus when an intense pulsed laser beam is focused into a gaseous medium. After optical breakdown occurs, the shock wave and the beam-absorbing plasma travel at several kilometers per second along the laser beam tube in the direction opposite to the beam incidence. These waves, which are known as the analogous combustion theory of the unique steady-state velocity given by the Chapman-Jouguet (CJ) condition, are laser-supported detonation (LSD) waves. ${ }^{3}$ At laser intensities higher than $10^{12}-10^{13} \mathrm{~W} / \mathrm{m}^{2}$, the propagation velocity of the ionization wave at the order of $10-100 \mathrm{~km} / \mathrm{s}$ is faster than that predicted by the CJ condition. ${ }^{4-8}$ In the combustion theory, it is possible for a detonation wave to move faster than the CJ state when the detonation wave is supported by some external forces (e.g., bullet, piston, and bends in pipe). ${ }^{9}$ In the LSD wave, the photoionization induced by the plasma UV radiation drives the fast-gas ionization wave with no gas-dynamics effect. ${ }^{5,10}$ Consequently, the structure and the mechanisms of the overdriven detonation are completely different between combustion and gas breakdown. In the Hugoniot analysis, the detonation waves in strong and weak overdriven (WO) states correspond to combustion and the discharge phenomena, respectively. ${ }^{3,11}$

For transition from the LSD regime to the isobaric heating (laser-supported combustion: LSC) regime, several studies reported that the termination condition of the LSD depends strongly on the ratio of its lateral expansion area to its front expansion area in a cylindrical laser tube. ${ }^{3,12}$ Nevertheless, few reports of the literature have described a theoretical or experimental study of the transition from the WO state to the CJ state. Fisher evaluated the threshold laser intensities of the transition using the rate equation of electron number density in the equilibrium condition assuming plasma parameters. ${ }^{6}$ Furthermore, most earlier experimental studies have obtained only one-dimensional emissions using old streak cameras. ${ }^{4}$ For the present study, we experimentally observed the WO state in the LSD regime using shadowgraph images obtained with a high-speed intensified CCD (ICCD) camera. Then, we investigated the transition from the WO state to the CJ state for a TEA $\mathrm{CO}_{2}$ laser beam in ambient air.

A transversely excited atmospheric $\mathrm{CO}_{2}$ pulse laser was used, as in our earlier studies. ${ }^{10,11}$ Its single-pulse energy is $10 \mathrm{~J}$. The incident laser beam is first reflected and forced using a $\mathrm{ZnSe}$ lens with a focal length of $70 \mathrm{~mm}$. An Al flattarget was set at $4.2 \mathrm{~mm}$ above the focal point. The pulse energy was measured at $10.3 \pm 0.2 \mathrm{~J}$ using a joule meter between the $\mathrm{ZnSe}$ focal lens and the target. To take shadowgraph images, a continuous wave diode laser with $1 \mathrm{~W}$ output power was used as a probe light. It projects the graph of a blast wave on a sensor of a high-speed ICCD camera (Ultra 8; DRS Technologies Inc.) that has resolution intensifiers of $512 \times 512$ pixels and can take eight frames in each operation. A maximum framing rate of 100 million frames per second with a minimum exposure time of $10 \mathrm{~ns}$ was set for this experiment.

Figure 1 presents a series of shadowgraph images in the time range of $14-84 \mathrm{~ns}, 102-172 \mathrm{~ns}$, and $250-3050 \mathrm{~ns}$. The $\mathrm{Al}$ flat-plate target was placed on the lower edge of the images; the laser beam was irradiated from the upper part. The single sequence consists of 8 photographs. The scale size of photographs is different in each sequence. From experiments, we observed the transition from the WO state to the CJ state in the LSD regime of $102-172 \mathrm{~ns}$ and the transition from the LSD regime to the LSC regime of $250-3050 \mathrm{~ns} .10^{8}$ and $2.5 \times 10^{6}$ frames per second were set in the first 16 frames and last 8 frames, respectively. In the final sequence, the shock wave traveled ahead of the ionization wave in the elapsed time at $2650 \mathrm{~ns}-3050 \mathrm{~ns}$. Because the shock wave propagates adiabatically, energy conversion from laser energy into kinetic energy stops. Thus, a transition from the LSD regime to the LSC regime was occurred at this timing. 


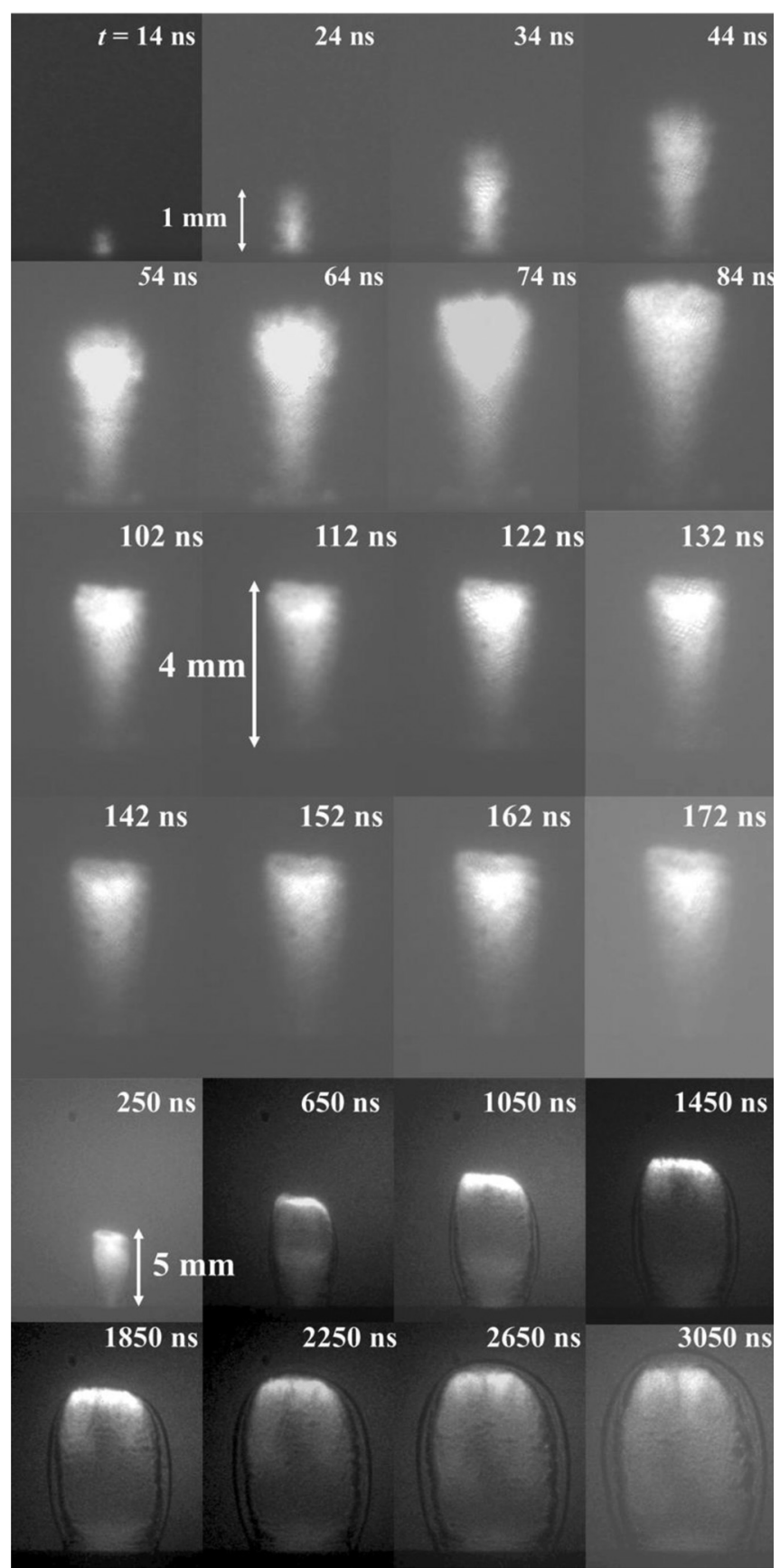

FIG. 1. Series of shadowgraph images: Nos. 1-8, time elapsed from 14 to 84 ns; No. 9-16, time elapsed from 102 to $172 \mathrm{~ns}$; and Nos. 17-24, time elapsed from $250 \mathrm{~ns}$ to $3050 \mathrm{~ns}$. Each sequence has a different photographic scale.

Figure 2 presents the displacement of the ionization wave and the shock wave from the target. The laser pulse shape, as measured using a photodetector, is also presented in Fig. 2. The pulse comprises a leading edge spike followed by an exponentially decaying tail. The full width at half maximum of the spike was $120 \pm 20 \mathrm{~ns}$. The tail decay constant was $1.15 \pm 0.05 \mu \mathrm{s}$. The typical square shaped beam cross sectional area of the TEA $\mathrm{CO}_{2}$ laser is $30 \mathrm{~mm} \times 30 \mathrm{~mm}$. The horizontal and vertical directions of the laser beam are the Gaussian and top-hat profiles, respectively. The value of beam quality factors $M^{2}$ was determined by measuring the beam width versus distance for the beam from the $\mathrm{CO}_{2}$ laser. ${ }^{13}$ The $M^{2}$ value of Gaussian and top-hat were 20 and 50,

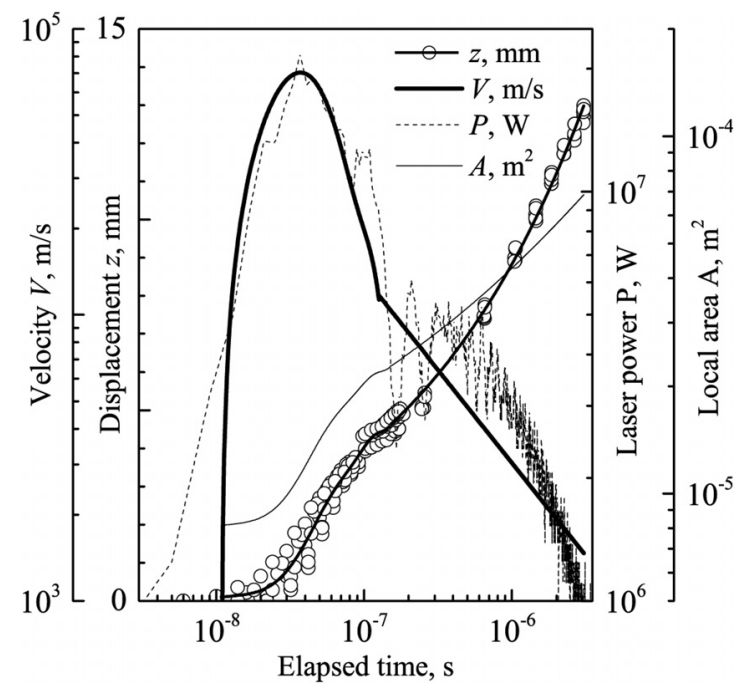

FIG. 2. Displacement of the ionization wave from the target, the ionization wave velocity, laser heating area at the ionization front, and laser pulse shape in terms of the elapsed time.

respectively. Using the $M^{2}$ value and $f$ number of focal lens, the square-shaped beam cross-sectional area at the focal point was calculated at $9.9 \times 10^{-7} \mathrm{~m}^{2}$. Assuming the laser focal shape as the quadrangular pyramid, we obtained the laser beam cross section as a function of the distance from the focal point as shown in Fig. 2. In Fig. 2, the LSD wave in the WO state, the first eight images in Fig. 1, was observed in the leading edge spike of the laser pulse shape.

Figure 3 presents the propagation velocity of the ionization wave as a function of the laser intensity. In Fig. 3, the results presented in Fig. 2 were compared with different gaseous forms, the laser wavelength, and the CJ velocity law. ${ }^{4,5,14}$ The ionization wave velocity was obtained from the experimental data and the derivative of fitted line for the displacement of the ionization wave in Fig. 2. The LSD velocity in the CJ condition for 1.06 and $10.6 \mu \mathrm{m}$ is described in Fig. 3. From Fig. 3, the transition threshold from the WO

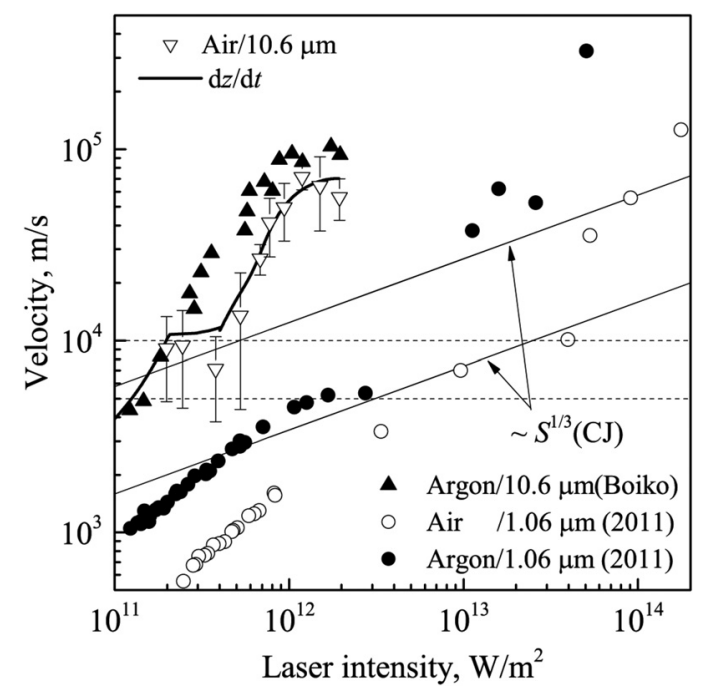

FIG. 3. Measurement results and the reference data of the ionization wave velocity in terms of laser intensity. ${ }^{4,14}$ The solid line on the experimental data was obtained from the derivative of the fitted line for the displacement of the ionization wave in Fig. 2. 
state to the CJ state for air and argon were $10 \mathrm{~km} / \mathrm{s}$ and $5 \mathrm{~km} / \mathrm{s}$, respectively. Those values are independent of the laser wavelength. In terms of the laser intensity, the transition thresholds for $10.6 \mu \mathrm{m}$ and $1.06 \mu \mathrm{m}$ laser wavelengths were $10^{11} \mathrm{~W} / \mathrm{m}^{2}$ and $10^{12}-10^{13} \mathrm{~W} / \mathrm{m}^{2}$, respectively. For both laser wavelengths, the transition threshold of the argon gaseous form was lower than that of air because the dissociation energy of molecules in air consumes the laser energy. To compare different laser experiments using the prediction of LSD termination, the dimensionless constant $C_{\mathrm{th}}^{\prime}$ is extendedly defined by the laser wavelength $\lambda$ and the laser peak power $P_{\text {peak. }}{ }^{12}$

$$
C_{t h}^{\prime}=r_{t h} S_{t h}\left(\frac{\lambda}{P_{\text {peak }}}\right)
$$

where $r_{\text {th }}$ and $S_{\text {th }}$ are the beam cross-sectional radius and the laser intensity at the transition from the WO state to the CJ state, respectively. Consequently, the dimensionless constant $C_{\mathrm{th}}^{\prime}$ for air remained approximately constant at unity, which is independent of the laser wavelength. Because the constant $C_{\mathrm{th}}^{\prime}$ is approximately $1 / 10$ in argon gas, the constant $C_{\mathrm{th}}^{\prime}$ might be a function of the structure of the atom and molecule, the ionization energy, and the nuclear charge.

To elucidate the transition mechanism from the WO state to the CJ state, the source of the seed electrons ahead of the ionization wave was evaluated. In the theory of fast-gas ionization, the photoionization attributable to plasma radiation ahead of the ionization wave plays an important role in generating the seed electron of the avalanche ionization. ${ }^{5}$ Besides, the intensity of plasma radiation increased proportionally to the laser irradiation intensity. ${ }^{15}$ The source term at the ionization wave front might affect the transition from the WO state to the CJ state because the laser intensity changes with the elapsed time. The rate equation of the electron density is

$$
\partial_{t} n_{e}=\left(\partial_{t} n_{e}\right)_{p h}+\nu_{i} n_{e}+D_{e} \Delta n_{e}-r n_{e}^{2},
$$

where $n_{\mathrm{e}}, \nu_{\mathrm{i}}, D_{\mathrm{e}}$, and $r$ stand for the electron density, the ionization frequency, the diffusion coefficient, and the recombination rate, respectively. The terms in the right-hand-side of Eq. (2) denote the photoionization attributable to the plasma UV radiation, the collisional ionization, the electron diffusion, and the radiative recombination, respectively. The photoionization and the electron diffusion should be considered for the source terms for the ionization wave front. To evaluate the increment of electron number density by photoionization $\left(\partial_{\mathrm{t}} n_{\mathrm{e}}\right)_{\mathrm{ph}}$, the total volumetric energy of the continuous plasma radiation (free-free and free-bound) $j_{\nu}$ in the frequency range corresponding to the photoionization can be expressed as ${ }^{16}$

$$
\int_{\nu_{i}}^{\infty} j_{\nu} d \nu=4.676 \times 10^{-14} \frac{n_{e}^{2}}{T_{e}^{1 / 2}} k_{B} T_{e} \exp \left(-\frac{h\left(\nu_{i}-\nu_{g}\right)}{k_{B} T_{e}}\right) .
$$

In this equation, $\nu_{\mathrm{i}}, \nu_{\mathrm{g}}, k_{\mathrm{B}}, T_{\mathrm{e}}$, and $h$ denote the ionization threshold frequency, the critical cut-off frequency, ${ }^{16}$ Boltzmann's constant, the electron temperature, and Planck's constant, respectively. Assuming all UV photon contributing to the electron increment, $\left(\partial_{\mathrm{t}} n_{\mathrm{e}}\right)_{\mathrm{ph}}$ can be estimated by the radiation power in Eq. (3) divided by the ionization energy of $\mathrm{O}_{2}$.
According to the spectroscopic data presented in a previous report ${ }^{14}$ and Eq. (3), $\left(\partial_{\mathrm{t}} n_{\mathrm{e}}\right)_{\mathrm{ph}}$ for $1.06 \mu \mathrm{m}$ laser wavelength in air and argon were on the order of $10^{31} \mathrm{~m}^{-3} \mathrm{~s}^{-1}$. However, the terms of electron diffusion $D_{\mathrm{e}} \Delta n_{\mathrm{e}}$ estimated by the characteristic diffusion length $\Lambda$ were evaluated at $10^{31} \mathrm{~m}^{-3} \mathrm{~s}^{-1}$ using the displacement $z$ and the beam cross-sectional radius $r^{17}$

$$
-D_{e} \Delta n_{e}=\frac{D_{e} n_{e}}{\Lambda^{2}}=D_{e} n_{e}\left[\left(\frac{\pi}{z}\right)^{2}+\left(\frac{2.405}{r}\right)^{2}\right] .
$$

At the transition from the WO state to the CJ state, the photoionization and the electron diffusion are in the comparable level because the ratio of the photoionization and the electron diffusion is unity. The result reveals that the source of the seed electrons ahead of the ionization wave regulates the transition from the WO state to the CJ state. After the transition, the photoionization is also the dominant process in the propagation of the LSD wave because the effect of photoionization on the seed electrons is relatively increased as time elapsed. The plasma volume rapidly increased proportionally to the plasma radiation and the inverse of the characteristic diffusion length, as presented in Fig. 2. However, $n_{\mathrm{e}}$ and $T_{\mathrm{e}}$ changed slightly with the elapsed time in a previous report of the literature. ${ }^{13}$ Thus, the ratio of the photoionization and the electron diffusion is greater than unity in the CJ state of the LSD regime.

The ionization degree, the plasma properties, and the plasma radiation affect the difference of the ionization velocity between the WO state and the CJ state. The results of this study demonstrated that the transition from the WO state to the CJ state can be elucidated by the source terms of the avalanche ionization at the ionization wave front, the plasma properties, and the plasma radiation. Further investigations of the plasma properties in the WO state are important to generalize the model.

This work was supported by JKA and its promotion funds from KEIRIN RACE.

\footnotetext{
${ }^{1}$ A. Sasoh, N. Urabe, S. S. M. Kim, and I.-S. Jeung, Appl. Phys. A 77, 349 (2003).

${ }^{2}$ L. J. Radziemski and D. A. Cremers, Laser-Induced Plasmas and Applications (CRC Press, New York, 1989).

${ }^{3}$ Y. P. Raizer, Sov. Phys. JETP 21, 1009 (1965).

${ }^{4}$ V. A. Boiko, V. A. Danilychev, B. N. Duvanov, V. D. Zvorykin, and I. V. Holin, Sov. J. Quantum Electron. 8, 134 (1978).

${ }^{5}$ V. I. Fisher, Sov. Phys. JETP 52, 1083 (1980).

${ }^{6}$ V. I. Fisher and V. M. Kharash, Sov. Phys. JETP 56, 1004 (1982).

${ }^{7}$ V. I. Fisher, Sov. Phys. Tech. Phys. 28, 1312 (1983).

${ }^{8}$ A. A. Ilyin, I. G. Nagorny, and O. A. Bukin, Appl. Phys. Lett. 96, 171501 (2010).

${ }^{9}$ J. Kasahara, T. Fujiwara, T. Endo, and T. Arai, AIAA J. 39, 1553 (2001).

${ }^{10}$ K. Shimamura, K. Komurasaki, J. A. Ofosu, and H. Koizumi, IEEE Trans. Plasma Sci. 42, 3121 (2014).

${ }^{11}$ K. Shimamura, J. A. Ofosu, K. Komurasaki, and H. Koizumi, Jpn J. Appl. Phys., Part 2 54, 5 (2015).

${ }^{12}$ K. Mori, K. Komurasaki, and Y. Arakawa, Appl. Phys. Lett. 88, 121502 (2006).

${ }^{13}$ A. E. Siegman, Proc. SPIE 1868, 2 (1993).

${ }^{14}$ K. Shimamura, K. Michigami, B. Wang, K. Komurasaki, and Y. Arakawa, AIP Conf. Proc. 1402, 326 (2011).

${ }^{15}$ O. A. Bukin, A. A. Il'in, Yu. N. Kul'chin, I. G. Nagornyi, A. N. Pavlov, and A. V. Bulanov, Quantum Electron. 36, 553 (2006).

${ }^{16}$ K. P. Horn, H. Wong, and D. Bershader, J. Plasma Phys. 1, 157 (1967).

${ }^{17}$ A. D. MacDonald, Microwave Breakdown in Gases (Wiley, New York, 1966).
} 